\title{
Hipoglicemia Hiperinsulinêmica Persistente Endógena no Adulto: Relato de Caso
}

\begin{abstract}
RESUMO
A hipoglicemia hiperinsulinêmica persistente endógena em adultos é, na maioria dos casos, causada por insulinoma. A Nesidioblastose, uma hiperinsulinemia funcional rara por hiperplasia das células beta do pâncreas, tem sido descrita principalmente em neonatos. Apresentamos o caso de uma paciente de 34 anos com quadro clínico-laboratorial compatível com hipoglicemia hiperinsulinêmica endógena (Glicemia jejum: $54 \mathrm{mg} / \mathrm{dl} /$ Valor de referência (VR): 60-99 $\mathrm{mg} / \mathrm{dl}$; Insulina sérica: 70,9 mcU/ml / VR: < 29,1 mcU/ml; e Peptídeo C: 7,1 ng/ml / VR: 1,1-5,0 ng/ml - simultâneos à glicemia). Foi aventada a hipótese de insulinoma. Em função da ausência de imagem característica aos exames radiológicos (ultra-som e tomografia de abdome), optou-se pela laparotomia exploradora, onde também não foi evidenciado tumor pancreático macroscopicamente. Os exames histopatológico e imuno-histoquímico evidenciaram hiperplasia de células beta, de segmento distal do pâncreas, compatível com nesidioblastose. A paciente evoluiu com estabilidade clínica por cerca de dois meses, quando se verificou recidiva das crises hipoglicêmicas, refratárias ao uso de Octreotide. Optou-se pela realização de "octreosan", que indicou nesidioblastose difusa, sendo procedida pancreatectomia parcial extensa. Seguiu-se o uso contínuo de Octreotide, com controle eficaz das crises hipoglicêmicas. Uma vez que esse é um diagnóstico raro no adulto, objetiva-se, nesse artigo, divulgar o manejo diagnóstico-terapêutico em casos de hipoglicemia hiperinsulinêmica endógena.
\end{abstract}

(Arq Bras Endocrinol Metab 2007;51/1:125-131)

Descritores: Hipoglicemia; Hiperinsulinemia; Células beta; Nesidioblastose

\begin{abstract}
Endogenous Persistent Hypoglicemia of Adult: Case Report.

Persistent Hyperinsulinemic Endogenous hypoglycemia in adults is, in most cases, due to Insulinoma. Nesidioblastosis, a peculiar functional hyperinsulinemia from hypertrophic beta cells, has been described mainly in newborns. This article describes a 34-year-old patient who presented hyperinsulinemic endogenous hypoglycemia clinical and laboratorial situation (Fasting glycemia: $54 \mathrm{mg} / \mathrm{dl}$ / Reference Interval (RI): 60-99 mg/dl; Serum insulin: $70.9 \mathrm{mcU} / \mathrm{ml} / \mathrm{RI}:<29.1$ $\mathrm{mcU} / \mathrm{ml}$; e C peptide: $7.1 \mathrm{ng} / \mathrm{ml} / \mathrm{Rl}: 1.1-5.0 \mathrm{ng} / \mathrm{ml}$ ). It was suspected Insulinoma. Because of the lack of typical images in radiologic exams (ultrasonography and computerized tomography) it had been decided to do laparotomy, but it was not found any macroscopic pancreatic tumor. Histological and histochemistry examination of a distal pancreatic segment showed alteration suitable to nesidioblastosis. The patient presented clinical stability during the next two months, however, after that, there was a recurrence of a hypoglycemia crisis, refractory to Octreotide administration. It was done "octreoscan", which showed expanded nesidioblastosis, being done extensive partial pancreatectomy. Octreotide was used again, with a good control of the hypoglycemia crisis. As it is an uncommon diagnosis in an adult, the objective of this article is to describe the diagnostic and therapeutic aspects in cases of hyperinsulinemic endogenous hypoglicemia.
\end{abstract} (Arq Bras Endocrinol Metab 2007;51/1:125-131)

Keywords: Hypoglycemia; Hiperinsulinemic; Beta cells; Nesidioblastosis apresentação de caso

\author{
RAQUel R. COSTA \\ FREDERICO F.R. MAIA \\ LEVIMAR R. ARaúJo
}

Departamento de Fisiologia e Clínica de Endocrinologia e Metabologia do Hospital Universitário São José, Faculdade de Ciências Médicas de Minas Gerais - FCMMG, Belo Horizonte, MG. 
$\mathrm{N}$ ESIDIOBLASTOSE, DERIVADO DO GREGO “nesidia”= ilhotas e "blastos" = germe, é um termo introduzido por Laidlaw, em 1938, para definir uma proliferação de células endócrinas originárias do epitélio ductular pancreático com conseqüiente hipersecreção inapropriada de insulina, sendo, desde então, conhecida como "hiperinsulinemia funcional por hiperplasia das células beta do pâncreas", "síndrome da dismaturidade das ilhotas pancreáticas" ou "síndrome de hipoglicemia hiperinsulinêmica persistente na infância - HHPI" (1).

A nesidioblastose representa a principal causa de hipoglicemia hiperinsulinêmica persistente em crianças (2), com manifestação inicial no período neonatal ou durante o primeiro ano de vida, sendo rara após essa faixa etária. Cerca de 0,5 a 10\% dos pacientes adultos com quadro clínico de hiperinsulinismo apresentam alterações em séries cirúrgicas, como hiperplasia, microadenomatose e nesidioblastose. O insulinoma é uma das causas mais comuns de hipoglicemia hiperinsulinêmica persistente endógena (HHPE) em adultos. Desde 1981, quando Harness e cols. (3) relataram a primeira série de casos de Nesidioblastose como causa de hipoglicemia em adultos, apenas 71 casos foram publicados em literatura indexada (4).

As apresentações clínicas da Nesidioblastose são amplas, podendo apresentar desde uma hipoglicemia leve e transitória até formas severas com obesidade, lesão cerebral e morte por uma hipoglicemia de difícil controle (5). A pancreatectomia (parcial ou sub-total, se nesidioblastose localizada ou difusa, respectivamente) é indicada como tratamento na maioria dos casos.

Apresentamos um caso de HHPE no adulto, sugestivo de insulinoma, porém não comprovado pelos métodos de imagem. A laparotomia e o exame imuno-histopatológico mostraram tratar-se de Nesidioblastose, um diagnóstico raro no adulto. Além de registrar esse caso raro, objetiva-se aqui discutir o manejo diagnóstico e terapêutico, bem como o prognóstico dessa doença.

\section{RELATO DO CASO}

Trata-se de LDML, sexo feminino, 34 anos, leucoderma, solteira, em acompanhamento endocrinológico desde 2001 para controle ponderal. A avaliação inicial mostrou índice de massa corpórea (IMC) de 23,3 $\mathrm{kg} / \mathrm{m}^{2}$ (VR: 18 a 24,9 $\mathrm{kg} / \mathrm{m}^{2}$ ). A paciente estava em tratamento para depressão, em uso diário de Fluoxetina, Tioridazina, Lorazepan e Clobazam, e asma persistente grave, em uso de Salmeterol, Fluticasona e Salbutamol. A história pregressa evidenciou exérese de
Astrocitoma Pilocítico Cerebelar aos quatro anos de idade. À história familiar, presença de irmã portadora de Diabetes Mellitus tipo 1 (DMl). Paciente informava ser não-tabagista e não-etilista.

Após aproximadamente três anos e meio de acompanhamento, a paciente iniciou com episódios de lipotímia, taquicardia, sudorese fria, sem perda de consciência segundo familiares. Foram achados baixos valores de glicemia capilar (GC) medida pela irmã diabética, que reconheceu os sinais de hipoglicemia (GC: $26 \mathrm{mg} / \mathrm{dL}$; VR: 4 5mg/dL). Houve melhora dos sintomas após ingestão de glicose. Sintomas semelhantes vinham ocorrendo há meses, porém menos intensos.

Diante do quadro clínico compatível com hipoglicemia, asma grave em uso de corticóide inalatório e depressão, foram aventadas as hipóteses de insulinoma, hipoglicemia factícia, insuficiência adrenal secundária, hipoglicemia auto-imune e deficiência de hormônio do crescimento.

A avaliação laboratorial mostrou glicemia em jejum: $54 \mathrm{mg} / \mathrm{dl}$ (VR: 60-99 mg/dl); insulina sérica: $70,9 \mathrm{mcU} / \mathrm{ml}(\mathrm{VR}:<29,1 \mathrm{mcU} / \mathrm{ml})$ e peptídeo $\mathrm{C}$ : $7,1 \mathrm{ng} / \mathrm{ml}$ (VR: $1,1-5,0 \mathrm{ng} / \mathrm{ml}$ ) (simultâneos à glicemia); cortisol sérico (às $10 \mathrm{hs}$ ): $11,9 \mathrm{mcg} / \mathrm{dl}$ (VR: 5,0-25,0 mcg/dl); auto-anticorpos anti-insulina: 0,8\% de ligação (VR: < 2,4\%); somatostatina: 0,3 nanog $/ \mathrm{ml}$ (VR: < 4,5 ng/ml).

A presença de hipoglicemia hiperinsulinêmica com peptídeo C elevado comprovou tratar-se de quadro clínico endógeno, reforçando, pois, a hipótese de insulinoma. Seguiu-se a avaliação por métodos de imagem, através de ultra-som (US) abdominal, que evidenciou pequena imagem hiperecogênica em cauda do pâncreas de caráter inconclusivo e achados semelhantes à tomografia (TC) abdominal. Em face da indefinição diagnóstica e manutenção do quadro clínico durante a investigação, foi realizada laparotomia, que não evidenciou massa tumoral visível ou palpável no pâncreas. O US trans-operatório também foi inconclusivo. Com base no quadro clínico-laboratorial, foi optado por exérese de parte da cauda do pâncreas para congelação durante a cirurgia, que demonstrou ausência de sinais de neoplasia e malignidade em linfonodos, hiperplasia de ilhotas de Langerhans associadas à proliferação ductular, formando complexos ducto-insulares compatíveis com Nesidioblastose. A comprovação pela imuno-histoquímica mostrou ilhotas hiperplásicas e células neuroendócrinas em grupos com acentuada quantidade de insulina e glucagon, compondo quadro característico de Nesidioblastose.

Durante o acompanhamento pós-cirúrgico, a paciente apresentou remissão clínica nos dois 
primeiros meses, voltando, porém, a apresentar episódios recorrentes graves de hipoglicemia após esse período, sendo iniciado tratamento com Octreotide. Não observou-se resposta satisfatória, sendo realizado "octreoscan", que sugeriu presença de hipertrofia de células beta difusamente no pâncreas. Decidiu-se, dessa forma, realizar uma segunda laparotomia. O pósoperatório imediato apresentou evolução desfavorável, com formação de coleção de secreção pancreática e sepse por Pseudomonas sp. A paciente necessitou de insulina exógena, uma vez que vinha mantendo-se em hiperglicemia. Suspeitou-se da sepse como provável causa, não descartando a possibilidade de insuficiência pancreática endógena após a pancreatectomia parcial extensa. Não houve sinais de insuficiência pancreática exógena. A paciente permaneceu internada por dois meses, apresentando progressiva melhora do quadro séptico sem resolução da hiperglicemia.

Após a alta hospitalar, a hiperglicemia se manteve, sendo necessário o uso regular de insulina NPH para controle, estabilizando o quadro durante seis meses. Após esse período, houve recidiva dos episódios de hipoglicemia, que se mantiveram mesmo com a suspensão da insulina NPH. Levantou-se a hipótese de presença de nesidioblastose no tecido mantido da cabeça do pâncreas. Iniciou-se o uso de octreotide, com remissão definitiva dos sintomas.

\section{DISCUSSÃO}

O caso relatado evidencia uma manifestação clínica típica de estado hipoglicêmico, com episódios de lipotímia, sudorese fria, taquicardia, baixos valores de glicemia capilar e melhora após ingestão de glicose, como descrito pela "Tríade de Whipple": glicemia $<55 \mathrm{mg} / \mathrm{dl} \mathrm{em}$ homens e $<45 \mathrm{mg} / \mathrm{dl} \mathrm{em}$ mulheres, sintomas compatíveis e melhora clínica após administração de glicose. Diante da evidência clínica de hipoglicemia, iniciou-se investigação laboratorial para definição diagnóstica e terapêutica nesse caso.

Com base na história de uso prolongado de corticóide inalatório (fluticasona $250 \mathrm{mcg} /$ dia) para tratamento de asma, uma das hipóteses foi de insuficiência adrenal (IA) secundária, devido à inibição de produção endógena de corticóide, o que foi afastado após dosagem sérica de cortisol dentro da normalidade, bem como de hormônio do crescimento, sabidamente hormônio contra-regulador da insulina, com potencial hiperglicemiante.

Em se tratando de paciente mulher, adultojovem, com sintomas depressivos e uma irmã diabética em uso de insulina, aventou-se à hipótese de hipoglicemia factícia. Nessas situações, observam-se níveis de peptídeo $\mathrm{C}$ desproporcionalmente baixos em relação à elevada concentração de insulina, uma vez que o peptídeo C é fisiologicamente secretado na proporção de 1:1 junto à insulina endógena (5). No caso em questão, foram evidenciados valores de insulina elevados (em relação à glicemia do momento) acompanhados por valores de Peptídeo $\mathrm{C}$ também elevados, achados não compatíveis com tal suspeita. Na hipoglicemia factícia por administração de sulfoniluréias, no entanto, há elevação tanto de insulina quanto de peptídeo $\mathrm{C}$, sendo a dosagem sérica de sulfoniluréias a melhor forma de estabelecer o diagnóstico diferencial. As hipoglicemias secundárias à doença auto-imune são formas pouco comuns em nosso meio. A presença de anti-corpos antiinsulina ou anti-receptor insulínico, associa-se ao aumento de insulina e pró-insulina, com supressão de peptídeo $\mathrm{C}$, o que não foi verificado nesse caso.

De acordo com o quadro descrito, a hipótese de insulinoma foi a mais provável, uma vez que esse tumor produtor de insulina é a causa mais conhecida de hipoglicemia hiperinsulinêmica em adultos. Pacientes com Insulinoma caracteristicamente apresentam-se com elevação dos níveis plasmáticos de insulina, peptídeo $\mathrm{C}$ e pró-insulina. A razão insulina/glicose geralmente é maior do que 0,30 , enquanto a relação insulina/próinsulina é de 1:1 (este último valor é de 5:1 em indivíduos normais e de 10:1 em indivíduos em uso de sulfoniluréias). Na presença de um Insulinoma, os níveis de insulina geralmente estão abaixo de $100 \mathrm{mU} / \mathrm{ml}$ (um pouco acima do limite superior da normalidade), sendo inapropriadamente alto na presença de hipoglicemia. Portanto, a terminologia hiperinsulinismo, usada freqüentemente, pode ser confusa, com a idéia de que há sempre um quadro de insulinemia elevada, o que não é verdade. De fato, a insulinemia está elevada para uma determinada concentração sérica de glicose, configurando uma verdadeira secreção inapropriada de insulina para as concentrações glicêmicas do momento (6), como visto no presente caso (insulina sérica: 70,9 $\mathrm{mcU} / \mathrm{ml}$ e razão insulina/glicose: 0,76 ).

Após definição do quadro de HHPE em adulto, a segunda etapa é identificar a localização do possível insulinoma. A ultra-sonografia, tomografia computadorizada ou ressonância magnética do abdome superior são as mais utilizadas, apesar de a sensibilidade desses exames de imagem ser baixa durante o pré-operatório (aproximadamente 50\%) devido ao reduzido tamanho habitual dos insulinomas (5). A US realizada via endoscópica melhora a sensibilidade para aproximadamente $80 \%$ (5). Através da arteriografia 
pancreática seletiva, consegue-se uma localização exata em $30 \%$ a $85 \%$ dos pacientes. Essa técnica é utilizada em poucos centros ainda no Brasil, não sendo acessível à maior parte da população. Há, ainda, a cintilografia com análogos da somatostatina (Octreoescan), cuja sensibilidade diagnóstica de $60 \%$ para insulinomas é menor do que em outros tumores pancreáticos.

A US trans-operatória tem sido considerada como o método mais sensível e específico, possibilitando a localização do insulinoma em mais de $90 \%$ dos pacientes. A palpação do pâncreas durante a cirurgia consegue identificar $80 \%-90 \%$ dos tumores. A combinação da palpação com a US trans-operatória pode propiciar uma sensibilidade próxima de $95 \%(7,8)$. No presente caso, a US abdominal, TC e US trans-operatória foram inconclusivas. O quadro clínico clássico indicou laparotomia, que possibilitou a confirmação diagnóstica pela análise histológica e imuno-histoquímica de nesidioblastose, uma doença obrigatoriamente considerada na hipoglicemia persistente neonatal, mas rara no adulto.

O critério histopatológico, apesar de existirem variantes, inclui ilhotas hipertrofiadas, ocasionalmente evidenciando células beta com pleomorfismo nuclear, complexos ducto-insulares e ilhotas neoformadas a partir dos ductos $(9,10)$, compatível com os achados no caso relatado. O posterior estudo imuno-histoquímico, que revelou presença de insulina e glucagon, corroborou tal diagnóstico. Achados na literatura mostram que pode ser freqüente a positividade para insulina, glucagon e somatostatina, havendo, porém, predomínio de secreção de insulina (11).

Esse caso reflete a dificuldade diagnóstica da nesidioblastose, dada sua semelhança com a apresentação do insulinoma, além da baixa acurácia dos métodos de imagem disponíveis. Na nesidioblastose, há inapropriada hipersecreção de insulina devido a uma proliferação de células endócrinas originárias do epitélio ductal pancreático, causando hipertrofia das ilhotas pancreáticas que, diferentemente do insulinoma, não formam lesão tumoral.

A fisiopatologia e a genética da Nesidioblastose em adultos não estão, ainda, tão bem definidos como em crianças. Já foram identificadas causas genéticas de HHPI, que dentre as mais comuns se encontra a forma autossômica recessiva devido à mutação no gene do receptor da sulfoniluréia (SURI), uma sub-unidade do canal de potássio ATP dependente localizado na membrana plasmática da célula beta que, sensível à glicose, regula a abertura de canais de cálcio que estimulam a secreção de insulina. A mutação altera a função do receptor que se torna incapaz de promover o fechamento do canal de cálcio, ocorrendo, dessa forma, secreção conti- nuada de insulina independente da glicemia. Foi descrita outra mutação no canal de potássio ATP dependente, na outra sub-unidade Kir6.2. Ambas as sub-unidades são codificadas por genes adjacentes no cromossoma 11 pl5.1 (12). Estão também descritos raros casos de formas de HHPI autossômicas dominantes, para os quais não se identificou ainda nenhuma mutação (13). Pacientes adultos com nesidioblastose não têm apresentado tais mutações, sendo necessários mais estudos genéticos nesse grupo de pacientes (14).

Achados atuais revelam alguma relação entre cirurgia bariátrica e hiperplasia das ilhotas pancreáticas, com conseqüente hipoglicemia. Nos últimos cinco anos, 40\% dos casos confirmados de Nesidioblastose ocorreram em pacientes que haviam sido submetidos a cirurgia bariátrica (15). Uma das hipóteses seria que, de alguma forma, o trânsito gastrointestinal intensamente alterado após a cirurgia bariátrica estaria estimulando essa proliferação das ilhotas pancreáticas. Uma outra possibilidade é a de pacientes obesos com resistência insulínica adaptativamente desenvolverem hipertrofia de células beta. Dessa forma, haveria hipoglicemia como conseqüência da melhora da sensibilidade à insulina resultante do peso perdido após a cirurgia. No entanto, não há associação comprovada entre nesidioblastose e perda de peso sem cirurgia bariátrica $(16,17)$.

Pode haver, também, alguma associação entre a nesidioblastose e o tumor neurológico apresentado previamente pela paciente em questão (Astrocitoma Pilocítico Cerebelar, aos quatro anos de idade). Há descrição de um caso com a tríade "nesidioblastose, neuroblastoma congênito e doença glomerulocistica" (18). Esta associação pode ser coincidente, mas pode haver um mecanismo patofisiológico comum.

A técnica de dosagem de insulina na veia hepática após infusão intra-arterial de Cálcio (ASVS) pode permitir uma ressecção guiada por gradiente, minimizando assim os riscos de ressecções excessivas ou, ao contrário, insuficientes. O paciente, idealmente, deve ser submetido a esse exame, que consiste na infusão de gluconato de cálcio $(0,025 \mathrm{mEq} / \mathrm{Kg})$, sucessivamente, nas artérias gastroduodenal, mesentérica superior e esplênica. Os níveis de insulina são, então, dosados em amostras coletadas da veia hepática direita antes e depois de cada infusão. O aumento, de duas vezes ou mais, dos níveis séricos de insulina indicam proliferação de células beta hiperfuncionantes na área vascular da artéria infundida (19-22). ASVS tem mostrado sensibilidade de aproximadamente $100 \%$ em casos de Insulinoma e Nesidioblastose $(23,24)$. No caso em questão, a paciente não pôde ser submetida a tal procedimento, uma vez que é alérgica a iodo. Dessa 
forma, a opção foi realizar o "octreoscan", que confirmou a suspeita de nesidoblastose difusa.

O tratamento da Nesidioblastose ainda causa algumas divergências. Em relação ao tratamento imediato, de suporte, há um consenso: glicose endovenosa, se necessário, com ou sem glucagon. Alguns autores defendem o tratamento clínico caso se consiga um controle das crises com tratamento medicamentoso, uma vez que há melhora clínica espontânea após 10 a 15 anos ou até mesmo depois de um ano de tratamento. Esta remissão poderia ocorrer devido a apoptose das células beta pancreáticas $(25,26)$. Outros autores defendem que o tratamento é fundamentalmente cirúrgico, sendo as respostas das medidas farmacológicas irregulares e parcialmente eficazes. Recomendam o tratamento medicamentoso apenas para tentar estabilizar o quadro clínico antes e após a cirurgia, ou mesmo quando esta é recusada pelo paciente ou contra-indicada (27).

O tratamento cirúrgico consiste em pancreatectomia parcial ou sub-total, de acordo com o tipo de Nesidioblastose, focal ou difusa, respectivamente. $\mathrm{Na}$ doença localizada, a ressecção menos extensa é apropriada. A forma focal representa cerca de $30 \%$ dos casos em pediatria (28), porém tem sido descrita como difusa ou multifocal, envolvendo quase todo o pâncreas, em adultos. Nesses casos, recomenda-se inicialmente uma pancreatectomia de $70-80 \%$, uso de octreotide em caso de hipoglicemia persistente no pósoperatório, e posterior ressecção mais extensa quando tais medidas falham $(29,30)$.

A pancreatectomia sub-total $(90 \%-95 \%)$ é indicada em todos os casos resistentes ao octreotide, segundo Filler e cols. (31). Porém, tal extensão cirúrgica associa-se a altas taxas de insuficiência pancreática endócrina e exócrina, devendo ser analisada com cautela. Nos casos confirmados de Nesidioblastose difusa, a pancreatectomia sub-total é necessária para remissão das hipoglicemias. Complicações a longo prazo, como diabetes mellitus insulino-dependente, secundário à pancreatectomia extensa, e desnutrição, secundária à deficiência de enzimas pancreáticas, devem ser acompanhadas e tratadas precocemente (32).

No presente caso, dada a refratariedade do tratamento clínico instituído (octreotide, $8 \mathrm{mg}$, SC, $8 / 8$ h), foi indicada nova cirurgia. No pós-operatório não foram observadas hipoglicemias; ao contrário, a paciente manteve hiperglicemia. Tal achado pode ter sido inicialmente devido à complicação por sepse que houve no período pós-cirúrgico inicial. Porém, a hiperglicemia se manteve mesmo após a resolução do quadro séptico e alta hospitalar, tendo a paciente que permanecer com o uso domiciliar de insulina $\mathrm{NPH}$.
De acordo com tal quadro, suspeitou-se de insuficiência endócrina de produção de insulina devido à extensão da pancreatectomia. Não foi observada insuficiência exócrina do pâncreas nesse momento. $\mathrm{O}$ diagnóstico de diabetes foi questionado após seis meses de uso de insulina, quando a paciente voltou a apresentar episódios de hipoglicemia. Interrompeu-se o uso da insulina NPH, com persistência das crises. Levantou-se a hipótese de recidiva devido a possível resíduo de ilhotas proliferadas na pequena parte do pâncreas que permaneceu (cabeça pancreática medindo 59 × 52 × 28 $\mathrm{mm})$. Tentou-se o tratamento com Octreotide $(0,05$ $\mathrm{mg}, \mathrm{SC}, 24 / 24 \mathrm{~h}$ ), estabilizando o quadro.

O Octreotide inibe a liberação de GH, TSH, glucagon, insulina, gastrina, VIP e outros hormônios de vários locais do trato gastrointestinal (33). Seu efeito é atribuído à supressão na liberação de insulina pelas células beta pancreáticas. As doses variam entre 5-20 $\mathrm{mcg} / \mathrm{kg} /$ dia IV ou SC. Altas doses podem agravar a hipoglicemia pela supressão do GH e glucagon. Em adultos, há relatos de aumento de incidência de cálculos biliares em pacientes tratados com esse medicamento, como visto no presente caso. Os resultados do uso de Octreotide são controversos, não havendo experiência suficiente de seu uso em adultos (34-39).

Existem outras opções de tratamento medicamentoso, mas também com efeitos colaterais importantes e eficácia questionável. Um dos fármacos mais utilizados é o Diazóxido (agonista dos canais K ATP), em doses orais divididas, atingindo o máximo de 15 $\mathrm{mg} / \mathrm{kg} /$ dia. Os efeitos colaterais principais são intolerância gástrica, retenção hídrica e hirsutismo, limitando o seu uso prolongado. A associação de um diurético tiazídico (em especial a hidroclorotiazida) ao Diazóxido age sinergicamente na abertura dos canais K ATP sensíveis e nos canais de $\mathrm{K}$ independentes de ATP, inibindo a secreção de insulina, além de, também, diminuir o efeito colateral de retenção hídrica (39). A Nifedipina também é utilizada com alguma freqüência, sobretudo em pacientes pediátricos (41), apesar de haver poucos relatos bem sucedidos. Sua ação parece basear-se na inibição das células beta através de mutações que alteram o funcionamento dos canais de cálcio dessas células (42).

Apesar de rara, a Nesidioblastose deve ser considerada frente a um caso de Hipoglicemia Hiperinsulinêmica Persistente no adulto. Na HHPI, a Nesidioblastose é a principal suspeita diagnóstica. Porém, na HHP não pediátrica, deve-se investigar, inicialmente, Insulinoma e hipoglicemia factícia, já que essas são as causas mais freqüentes. Após investigadas tais hipóteses diagnósticas, deve-se considerar a presença de 
Nesidioblastose, sendo a forma difusa sua manifestação mais comum. O tratamento definitivo é difícil, havendo alta probabilidade de recidivas após pancreatectomias parciais. Complicações como diabetes e desnutrição devem ser esperadas e abordadas de maneira adequada.

\section{REFERÊNCIAS}

1. Laidlaw GF. Nesidioblastoma, the islet tumor of the pancreas. Am J Pathol 1938; 14:125-34.

2. De Londay P, Poggi-Travert F, Fournet JC. Clinical features of 52 neonates with hyperinsulinism. N Engl J Med 1999;340:1169-75.

3. Harness JK, Geelhoed GW, Thompson NW. Nesidioblastosis in adults. A surgical dilemma. Arch Surg 1981;116:575-80.

4. Jabri AL, Bayard C. Nesidioblastosis associated with hyperinsulinemic hypoglicemia in adults: review of the literature. Eur J Intern Med 2004;15:407-10.

5. Vilar L. Endocrinologia Clínica. 2a ed. Rio de Janeiro: Editora Médica e Científica, 2001. pp. 688-700.

6. Ravel R. Laboratório Clínico. 6a ed. Rio de Janeiro: Guanabara Koogan, 1997. pp. 408-12.

7. Harrison TS, Fajans SS, Floyd JC. Prevalence of diffuse pancreatic beta islet cell disease with hyperinsulinism: problems in recognition and management. World J Surg 1984;8:583-9.

8. Hashimoto LA, Walsh RM. Preoperative localization insulinomas is nor necessary. J Am Coll Surg 1999;189:368-73.

9. Klaus MD, Bruno MD. Nesidioblastosis: An old term and a new understanding. World J Surg 2004;28:1227-30.

10. Reineckeluthge AKF, Koppel G. The molecular basis of persistent hyperinsulinemic hypoglycemia of infancy and its pathologic substrates. Virchows Arch 2000;436:1-5.

11. Gould VE, Chejfec G, Paloyan E, Lawence AM. Adult nesidiodysplasia. Semin Pathol 1984;1:43-53.

12. Nertorowicz A, Anagaki N, Gonoi T. A nonsense mutation in the inward rectifier potassium channel gene, kir 6.2is associated with familial hyperinsulinemism. Diabetes 1997;46:1743-8.

13. Thornton PS, Satinsmith MS, Herold K. Familial hyperinsulinism with apparent autossomal dominant inheritance-clinical and genetic differences from the autossomal recessive variant. J Pediatr 1998;132:9-14.

14. Service FJ, Nath N, Thompson GB. Noninsulinoma pancreatogenous hypoglycemia: a novel syndrome of hyperinsulinemic hypoglycemia in adults independent of mutation in Kir6.2 and SUR 1 genes. J Clin Endocrinol Metab 1999;84:1582-9.

15. Steinbrook R. Surgery for severe obesity. N Engl J Med 2004;350:1075-9.

16. Cummings DE. Gastric bypass and nesidioblastosis - too much of a good thing for islets? N Engl J Med 2005;3:300-2.

17. Service FJ, Thopson GB, Andrews JC, Collazo-Clavel ML, Lloyd RV. Hyperinsulinemic Hypoglycemia with Nesidoblastosis after gastric-bypass surgery. N Engl J Med 2005;3:249-54.

18. Bulum A, Sarici SU, Soyer OU, Tedsam O, Yurdakok M, Caglar M. The triad of nesidioblastosis, congenital neuroblastoma and glomerulocystic disease of the newborn: a case report. Turk J Pediatr 2005;47(3):298-302.

19. Shea D, Rohrer-Theurs AW, Lynn JA, Jackson JE, Bloom SR. Localization of insulinomas by selective intrarterial calcium injection. J Clin Endocrinol Metab 1996;81:1623-7.

20. Doppman JL, Miller DL, Chang R, Shawker TH. Insulinomas: localization with selective intrarterial injection of calcium. Radiology 1991;178:237-41.

21. Doppman JL, Chang R, Fraker DL, Norton JA. Localization of insulinomas to regions of the pancreas by intrarterial stmulation with calcium. Ann Intern Med 1995;123:269-73.

22. Lee WL, Won JGS, Chiang JH, Hwang JI. Selective intra-arterial calcium injection in the investigation of adult Nesidioblastosis: a case report. Diabetes Med 1997;14:985-88.
23. Thompson GB, Service FJ, Andrews JC. Noninsulinoma pancreatogenous hypoglycemia syndrome: an update in 10 surgically treated patients. Surgery 2000;128(6):937-44.

24. Brandle M, Pfammatter $T$, Spinas GA, Lehmann R, Schmid C. Assessment of selective arterial calcium stimulation and hepatic venous sampling to localize insulin-secreting tumours. Clin Endocrinol 2001;55(3):357-62.

25. de Lonlay P. Persistent hyperinsulinemic hypoglycemia. Orphanet Encyclopedia 2003;june.

26. Kassen SA, Ariel I, Thornton PS, Scheimberg I, Glaser B. Beta-cell proliferation and apoptosis in the developing normal human pancreas and in hyperinsulinism of infancy. Diabetes 2000;49:1325-33.

27. Branco VA, Santos RM, Cipriano MA, Tralhão G, Otero M, Moura JA, et al. Adult Nesidioblastosis. Act Med Port 2003; $16: 465-70$

28. Dubois J, Brunelle F, Touati G, Sebag G, Nuttin C, Thach T. Hyperinsulinism in children: diagnostic value of pancreatic venous sampling correlated with clinical, pathological and surgical outcome in 25 cases. Pediat Radiol 1995;25:512-6.

29. Carlson T, Eckhauser ML, Debaz B, Khiyami A, Park CH. Nesidioblastosis in an adult: an illustrative case and collective review. Am J Gastroenterol 1987;82(6):566-71.

30. Rinker RD, Friday F, Aydin F, Jaffe BM, Lambiase L. Adult nesidiblastosis: a case report and review of the literature. Dig Dis Sci 1998;43(8):1784-90.

31. Filler RM, Weinberg MJ, Cutz E, Wesson DE, Ehrlich RM. Current status of pancreatectomy for persistent idiopathic neonatal hypoglicemia due to islet cell dysplasia. J Pediatr Surg 1991;26:60-75.

32. Debeney LP, Travert PF, Fournet JC. Clinical features of 52 neonates with hyperinsulinism. N Engl J Med 1999;340: 1169-75.

33. Ferraz DP, Almeida MS, Mello B F. Uso de octreotide na hipoglicemia hiperinsulinêmica persistente infantil. Arq Bras Endocrinol Metab 2005;49:460-7.

34. Wal BC, Krijger RR, Herder WW. Adult hyperinsulinemic hypoglycemia not caused by an insulinoma: a report of two cases, Virchows Arch 2000;436:481-6.

35. Mozell EJ, Woltering EA, O'Dorisio TM. Adult onset nesidioblastosis: response of glucose, insulin, and secondary peptides to therapy with Sandostatin. Am J Gastroenterol 1990;85(2): 181-8.

36. Fuller PJ, Ehrlich AR, Susil B, Zeimer H. Insulin gene expression in adult onset nesidioblastosis, Clin Endocrinol 1997;47(2):245-50.

37. Garcia JP, Franca T, Pedroso C, Cardoso C, Cid MO. Nesidioblastosis in the adult surgical management. HPB Surg 1997;10(4):245-50.

38. Lecube A, Obiols G, Ramos I, Gemar E. Hyperinsulinemic hypoglicemia and nesidioblastosis in adults. An exceptional disease, Med Clin 2001;116(6):238-9.

39. Vara AC, Bereciartua AF, Munoz MA, Gonzales G. Focal and diffuse nesidioblastosis. Med Clin 2002;119(5):199.

40. Witteles RM, Straus FH, Sugg SL, Koka MR, Costa EA, Kaplan EL. Adult-onset nesidioblastosis causing hypoglycemia: an important clinical entity and continuing treatment dilemma. Arch Surg 2001;136(6):656-63.

41. Dunne MJ, Kane C, Shepherd RM. Familial persistent hyperinsulinemic hypoglycaemia of infancy and mutations in the sulfonylurea receptor. N Engl J Med 1997;336:703-6.

42. Lindley KJ, Dunne MJ, Kane C. Ionic control of beta cell function in nesidioblastosis. A possible therapeutic role for calcium channel blockade. Arch Dis Child 1996;74(5):373-8.

Endereço para correspondência:

Raquel Rangel Costa

Rua Oriente 685, ap. 303

30220-270 Belo Horizonte, MG

E-mail: raquelrangel@ig.com.br 